\title{
Focal facial dermal dysplasia type III
}

INSERM

\section{Source}

INSERM. (1999). Orphanet: an online rare disease and orphan drug data base. Focalfacial dermal dysplasia type III. ORPHA:1807

Focal facial dermal dysplasia type III (FFDD3) is a rare focal facial dermal dysplasia (FFDD; see this term), characterized primarily by cong enital bitemporal scar-like depressions and a typical, but variable facial dysmorphism, which may include distichiasis (upper lids) or lacking eyelashes, slanted eyebrows and a flattened and/or bulbous nasal tip and other features such as a low frontal hairline, sparse hair, redundant skin, epicanthal folds, lowset dysplastic ears, blepharitis and conjunctivitis. 